\title{
Surface Reconstruction of a Three-Dimensional Ultrasonic Flaw
}

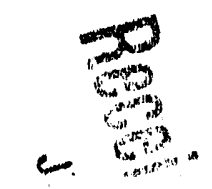

Lat S. Koo

Idaho National Engineering Laboratory

EGG-M--91631

EG\&G Idaho, Inc.

P.O. Box 1625

Idaho Falls, ID 83415-2209

LE92 018029

U.S.A.

\section{Summary}

In three-dimensional inverse scattering problems, the reconstruction of a solid scatterer is often difficult, if not impossible, and computationally expensive due to the dimensionality. To obtain only the geometrical information, a surface reconstruction algorithm is naturally more desirable since no additional knowledge can be gained from doing the solid reconstruction and the computation is reduced to two dimensions. With the application of the first Born approximation, this paper proposes a simple surface reconstruction technique for a three-dimensional target. In general, this method is ill-posed. However, the numerical instability part of the ill-posedness is removable when the surface has a two-fold symmetry with respect to a plane. To demonstrate this approach, three analytical examples are shown.

\section{Introduction}

Reconstruction of the geometry of an embedded flaw has been one of the major areas in nondestructive evaluation (NDE) research. Some of the algorithms published in the last decade were based on the elastic wave inverse (first) Bom approximation, which is essentially a low frequency approximation to an isolated weak scatterer in an otherwise isotropic and homogeneous medium whose material properties are similar to the host's [1,2]. A key factor in the development of these inverse algorithms is the spatial-frequency function "shape factor" that appears in the formulation of the inverse Born approximation. This shape factor embodies all information about the size, shape, and orientation of the scatterer and, therefore, one can develop inverse procedures to extract the geometry in terms of a characteristic function (which has the value 1 inside the scatterer and 0 outside). The direct approach to recovering this characteristic function requires three-dimensional information in the spatial-frequency domain [3], which is computationally expensive. However, the basic equation of the general three-dimensional inverse Born approximation can be further cast to form a set of equations for the surface of a three-dimensional flaw. As a consequence, one can develop a "surface reconstruction" algorithm based upon these equations. Since the surface of a three-dimensional flaw is two-dimensional, the algorithm requires only knowledge of a two-dimensional hyper-plane of the shape factor instead of a complete three-dimensional domain, which reduces the dimensionality by one. The present

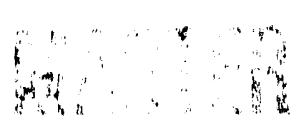
algorithm can be reduced to a special case that was suggested by R. M. Lewis [4]. For flaws having very dissimilar material properties (strong scatterers), the aforementioned methods may still be applicable since studies have shown that the inverse Born approximation is useful not onlX in IMITEO 
sizing weak scatterers but also strong ones [5]. This was recently shown when Chen [6] applied the elastodynamic ray theory to an arbitrary void in an isotropic and homogeneous medium and cound that the inverse Born approximation accurately predicted both the front surface echo and the constant term in the asymptotic expansion of the impulse responset as long as the host material has a Poisson ratio of $1 / 3$. Theretore, for a host material having a Poisson ratio about $1 / 3$ (this is true for most structural materials), the early-arriving portion of the response signal is expected to agree with the Born prediction.

The next section describes the theory behind the surface reconstruttion method (a more detailed discussion of this technique will be reported in a future publication [7]). Then analytical examples of reconstruction of three scatterers are given. After the examples, the reconstruction algorithms for a finite spatial-frequency bandwidth are given.

\section{Theory}

Based on the inverse (first) Bom approximation, for a pulse-echo test with a longitudinal impulsive incident wave, the longitudinal scattering amplitude $A$ can be written as $[1,5]$

$$
A=\frac{k^{2}}{4 \pi} B S(q)
$$

where $\mathrm{k}$ is the longindinal wave number and $\mathrm{B}$, which is dependent upon material properties of both the flaw and the host, is

$$
B=\frac{\delta \rho}{\rho}+\frac{\delta \lambda+2 \delta \mu}{\lambda+2 \mu}
$$

where $\lambda, \mu$, and $\rho$ are respectively the Lamé elastic constants and the mass density of the host and $\delta \lambda=\lambda_{\text {flaw }}-\lambda$, etc. In this paper, a bold-typed letter denotes a vector (e.g. q). In (1), $q=|q|=$ $2 \mathrm{k}$ where $|\mathrm{q}|$ denotes the magnitude of $\mathrm{q} . \mathrm{S}(\mathrm{q})$ is the shape factor, which embodies all the information about the size, shape, and orientation of the scatterer and forms a Fourier transform pair with the three-dimensional characteristic function $\gamma^{\prime} \mathbf{x}$ ) (which has the value 1 inside the scatterer and 0 outside) [5]

$$
\begin{aligned}
S(q) & =\int_{-\infty}^{\infty} \int_{-\infty}^{\infty} \int_{-\infty}^{\infty} d V(x) \gamma(x) e^{i q \cdot x} \\
& =\int_{-\infty}^{\infty} \int_{-\infty}^{\infty} d x d y e^{i\left(q_{x} x+q_{y} y\right)} \int_{-\infty}^{\infty} d z \gamma(x) e^{i q_{z} z} .
\end{aligned}
$$

In (3), $\mathrm{aV}(\mathbf{x})$ denotes the differential volumetric element with the position vector $x=(x, y, z)$ in the infinite spatial domain. From (1), $S(q)$ can be expressed as 


$$
S(q)=C \frac{A}{k^{2}} .
$$

Here the proportionality $C=4 \pi / B$ is a function of material properties only. Once $S(q)$ is known, $\gamma(x)$ can be obtained by applying the inverse-Fourier transformation to (3). In (3), when one isolates $\gamma(x)$ along the $z$-direction, then

$$
S(q)=\int_{-\infty}^{\infty} \int_{-\infty}^{\infty} d x d y e^{i\left(q_{x} x+q_{y} y\right)} g\left(u, v ; q_{z}\right)
$$

where $u(x, y)$ and $v(x, y)$ are the upper and lower surfaces, respectively, of the flaw along the $z$ axis in the spatial domain (Fig. 1). Obviously, $u$ and $v$ depend on which plane is chosen as $z=$ constant. For convenience, sometimes the coordinate is designated such that $\mathrm{z}=0$ cuts through the centroid of the flaw and separates $u$ and $v$. These two surface functions are assumed to be singlevalued. In (5), the unknown $g\left(u, v ; q_{z}\right)$ is a nonlinear fui.ction of $u$ and $v$ with a constant $q_{z}$

$$
g\left(u, v ; q_{z}\right)=\int_{v(x, y)}^{u(x, y)} d z e^{i q_{z} z} .
$$

Notice that $q_{z}=$ constant is the only hyper-plane in the shape factor domain (q) we will extract information from. In [4], $\mathrm{q}_{z}=0$ was used, here $\mathrm{q}_{z} \neq 0$ is assumed. If $\mathrm{q}_{\mathrm{z}}=0, \mathrm{~g}(\mathrm{u}, \mathrm{v} ; 0)$ becomes the thickness function of the scatterer along the $z$-direction and one must seek a second equation in order to resolve $u$ and $v$. To acquire the second equation, Lewis [4] differentiated (8) with respect to $\mathrm{q}_{\mathrm{z}}$ and then set $\mathrm{q}_{\mathrm{z}}=0$ while the present proposed technique uses a plane $\mathrm{q}_{\mathrm{z}} \neq 0$ and resolves both $\mathrm{u}$ and $\mathrm{v}$ from (8) alone without any differentiation. In the following discussion, the numerical instability is shown to be related to the nonsymmetry of the scatterer surface and thus can be eliminated when symmetry is preserit. A two-dimensional version of the above nonlinear Fredholm integral equation of the first kind (5) was considered by both Kogan and Lopes [8] and Vogel [9]. While Kogan and Lopes used a guess-and-iterate scheme to calculate $u$ and $v$ for a double-rectangular box with simulated geometric scattering data, Vogel applied a nonlinear opuimization scheme similar to the Levenberg-Marquardt algorithm [9] to recover $u$ and $v$ for a peanut-nut-shaped flaw with simulated scattering data. In the present paper, we will demonstrate a much simpler algorithm derived from (5) and (6) for a three-dimensional target. When $u$ and $v$ are

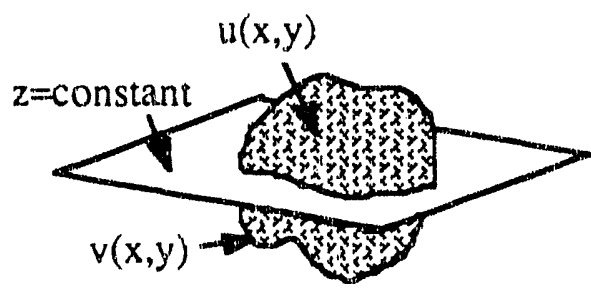

Figure 1. Defining the upper, $u(x, y)$, and lower, $v(x, y)$, surfaces. 
symmetrical to each other, the proposed algorithm becomes numerically stable. Since $\mathrm{g}\left(\mathrm{u}, \mathrm{v} ; \mathrm{q}_{2}\right)$ forms a two-dimensional Fourier transform pair with $S(q), g\left(u, v ; q_{z}\right)$ can be also written as

$$
g\left(u, v ; q_{z}\right)=\frac{1}{(2 \pi)^{2}} \int_{-\infty}^{\infty} \int_{-\infty}^{\infty} d q_{x} d q_{y} s(q) e^{-i\left(q_{x} x+q_{y} y\right)} .
$$

Notice that the right hand side of (7) is a measurable quantity. For convenience, this quantity will be rendered as $d\left(x, y ; q_{z}\right)$ hereafter. That is,

$$
g\left(u, v ; q_{z}\right)=d\left(x, y ; q_{z}\right)
$$

where $u$ and $v$ are functions of $x$ and $y$. By further evaluating the integral in (6), we find

$$
\begin{aligned}
& u=\frac{1}{q_{z}}\left[\tan ^{-1} \frac{\operatorname{Im}(d)}{\operatorname{Re}(d)}+\sin ^{-1} \frac{q_{z}|d|}{2}+N_{u} \pi\right] \\
& v=\frac{1}{q_{z}}\left[\tan ^{-1} \frac{\operatorname{Im}(d)}{\operatorname{Re}(d)}-\sin ^{-1} \frac{q_{z}|d|}{2}+N_{v} \pi\right]
\end{aligned}
$$

where $\operatorname{Re}(d)$ and $\operatorname{Im}(d)$ denote the real and imaginary parts of $d\left(x, y ; q_{z}\right)$, respectively, $q_{z} \neq 0$, and $N_{u}, N_{v}=0, \pm 1, \pm 2, \pm 3, \ldots$. Contrary to the original surfaces, the above $u$ and $v$, seemingly, are functions not only of $x$ and $y$, but also of $q_{z}$ and the integers $N_{u}$ and $N_{v}$. However, from the three examples which will be shown in the following section, the $\mathrm{q}_{z}$ term is cancelled by the first two terms in both (9a) and (9b) such that it can be arbitrary and the integers, $N_{u}$ and $N_{v}$, are always zero. The ill-posedness in (6) is clearly demonstrated in the first terms in (9). However, when u and $v$ are syrnmetrical to each other with respect to $z=$ constant, these terms becorne zero. That is, if $u=-v$, then $\operatorname{Im}(d)=0$ and $(9)$ becomes a numerically stable algorithm

$$
\begin{aligned}
& \mathrm{u}=\frac{1}{\mathrm{q}_{\mathrm{z}}}\left[\sin ^{-1}-\frac{\mathrm{q}_{\mathrm{z}} \mathrm{ld} \mid}{2}+\mathrm{N} \pi\right] \\
& \mathrm{v}=-\mathrm{u}
\end{aligned}
$$

where $\mathrm{N}=0, \pm 1, \pm 2, \pm 3, \ldots$.

\section{Analycical examples}

For any finite right cylinder with the major axis aligned along the $z$-direction, its three-dimensional characteristic function $\gamma(\mathbf{x})$ becomes separable as

$$
\gamma(x)=\gamma(z) \gamma(x, y)
$$

Substituting (11) into (3) and then applying the result into ( 7 ), the measurable quantity $\mathrm{d}\left(\mathrm{x}, \mathrm{y} ; \mathrm{q}_{2}\right)$ becomes

$$
d\left(x, y ; q_{z}\right)=\int_{-\infty}^{\infty} \gamma(z) e^{-i q_{z} z} d z \gamma(x, y)
$$




$$
=\frac{2 \sin \left(q_{z} a_{z}\right)}{q_{z}} e^{i q_{z}\left(1-p_{z}\right) a_{z}} \gamma(x, y)
$$

where $-p_{z} a_{z}$ is the distance between the bottom surface $v(x, y)$ and the origin with $2 a_{z}$ being the axial length. As a consequence of (12) and (9) together with $N_{u}=N_{v}=0$, the top and bottom surfaces can be recovered exactly as

$$
\begin{aligned}
& u(x, y)=\left(2-p_{z}\right) a_{z} \gamma(x, y) \\
& v(x, y)=-p_{z} a_{z} \gamma(x, y) .
\end{aligned}
$$

The expression of the two-dimensional characteristic function $\gamma(x, y)$ depends upon not only the coordinates on the $x-y$ plane but also on the location of the origin, which can be anywhere inside or outside the scatterer. Examples of a rectangular parallelepiped (Fig. 2a) and a finite right circular cylinder (Fig. 2b) given below will verify the above results (13). In addition, equations (10) will be tested on a third scatterer: a spheroid (Fig. 2c). Only results are given here and all the details of the calculations will be reported in [7].

\section{Rectangular Parallelepiped}

The semi-lengths of this target are assumed to be $\left(a_{x}, a_{y}, a_{z}\right)$ along the Cartesian $(x, y, z)$ directions respectively (Fig. 2a). The origin of the coordinate is assumed to be $\left(p_{x} a_{x}, p_{y} a_{y}, p_{z} a_{z}\right)$ away from the sides on the negative $(x, y, z)$ axes respectively. Equivalently, the origin is $\left(\left(2-p_{x}\right) a_{x},\left(2-p_{y}\right) a_{y}\right.$, $\left.\left(2-p_{z}\right) a_{z}\right)$ away from the sides on the positive $(x, y, z)$ axes. The ratios $p_{x}, p_{y}$, and $p_{z}$ are all real numbers and $0 \leq p_{x}, p_{y}, p_{z} \leq 2$. Correspondingly, the shape factor (3) becomes

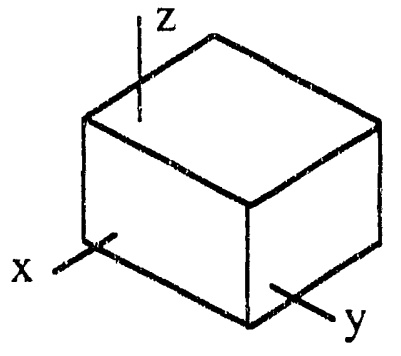

(a)

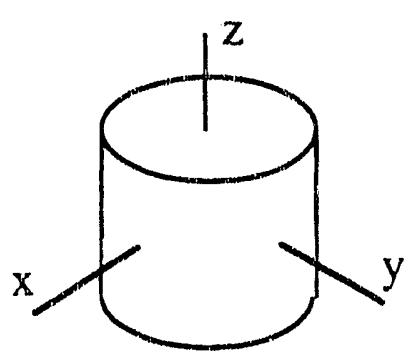

(b)

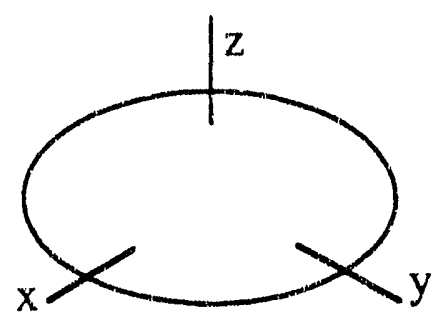

(c)

Figure 2. Three scatterers: (a) a rectangular parallelepiped, (b) a finite right circular cylinder, and (c) a spheroid with circular symmetry on the $x-y$ plane. 


$$
S(q)=\frac{8}{q_{x} q_{y} q_{z}} \sin \left(q_{x} a_{x}\right) \sin \left(q_{y} a_{y}\right) \sin \left(q_{z} a_{z}\right) e^{i q_{x}\left(1-p_{x}\right) a_{x}} e^{i q_{y}\left(1-p_{y}\right) a_{y}} e^{i q_{z}\left(1-p_{z}\right) a_{z}}
$$

Substituting (14) into (8), $d$ is

$$
\begin{aligned}
& \mathrm{d}\left(\mathrm{x}, \mathrm{y} ; \mathrm{q}_{\mathrm{z}}\right)=\frac{2 \sin \left(\mathrm{q}_{\mathrm{z}} \mathrm{a}_{\mathrm{z}}\right)}{\mathrm{q}_{\mathrm{z}}} \mathrm{e}^{\mathrm{i} \mathrm{q}_{\mathrm{z}}\left(1-\mathrm{p}_{\mathrm{z}}\right) \mathrm{a}_{\mathrm{z}}} \cdot\left[\mathrm{H}\left(\mathrm{x}+\mathrm{p}_{\mathrm{x}} \mathrm{a}_{\mathrm{x}}\right)-\mathrm{H}\left(\mathrm{x}-\left(2-\mathrm{p}_{\mathrm{x}}\right) \mathrm{a}_{\mathrm{x}}\right)\right] \\
& \text {. [H( } \left.\left(y+p_{y} a_{y}\right)-H\left(y-\left(2-p_{y}\right) a_{y}\right)\right]
\end{aligned}
$$

where $H$ is the Heaviside unit function. Consequently, from (9) with $N_{u}=N_{v}=0$,

$$
\begin{aligned}
u(x, y)= & \left(2-p_{z}\right) a_{z}\left[H\left(x+p_{x} a_{x}\right)-H\left(x-\left(2-p_{x}\right) a_{x}\right)\right] \\
& \cdot\left[H\left(y+p_{y} a_{y}\right)-H\left(y-\left(2-p_{y}\right) a_{y}\right)\right] \\
v(x, y)= & -p_{z^{2}} a_{z}\left[H\left(x+p_{x} a_{x}\right)-H\left(x-\left(2-p_{x}\right) a_{x}\right)\right] \\
& {\left[H\left(y+p_{y} a_{y}\right)-H\left(y-\left(2-p_{y}\right) a_{y}\right)\right] }
\end{aligned}
$$

which are exactly the upper and lower surfaces of the rectangular parallelepiped.

\section{Finite Right Circular Cylinder}

The major axis of the cylinder is aligned with the $z$ axis and the origin is placed $-\mathrm{p}_{z} \mathrm{a}_{z}$ away from the bottom surface (Fig. 2.b). Its radius and semi-length are assumed to be $\left(a_{x}, a_{2}\right)$ respectively. Correspondingly, the shape factor (3) becomes

$$
S(q)=\frac{4 \pi a_{x} \sin \left(q_{z} a_{z}\right) J_{1}\left(b a_{x}\right)}{b q_{z}} e^{i q_{z}\left(1-p_{z}\right) a_{z}}
$$

where $b=\sqrt{\left(q_{x}\right)^{2}+\left(q_{y}\right)^{2}}$ and $J_{1}$ is the Bessel function of the first kind and first order. Substituting (17) into (8), $d$ is

$$
\mathrm{d}\left(\mathrm{x}, \mathrm{y} ; \mathrm{q}_{\mathrm{z}}\right)=\frac{2 \sin \left(\mathrm{q}_{z} \mathrm{a}_{\mathrm{z}}\right)}{\mathrm{q}_{\mathrm{z}}} \mathrm{e}^{\mathrm{i} \mathrm{q}_{\mathrm{z}}\left(1-\mathrm{p}_{\mathrm{z}}\right) \mathrm{a}_{\mathrm{z}}} \mathrm{H}\left(\mathrm{a}_{\mathrm{x}}-\mathrm{r}\right)
$$

where $r$ is the radial length on the $x-y$ plane. Consequently, from (9) with $N_{u}=N_{v}=0$,

$$
\begin{aligned}
& u(x, y)=\left(2-p_{z}\right) a_{z} H\left(a_{x}-r\right) \\
& v(x, y)=-p_{z} a_{z} H\left(a_{x}-r\right)
\end{aligned}
$$

which are exactly the upper and lower surfaces of the right circular cylinder.

\section{Spheroid}

The spheroid is centered at the origin with its semi-axes $\left(a_{x}, a_{x}, a_{z}\right)$ along the $(x, y, z)$ directions respectively (Fig. 2c). Correspondingly, the shape factor (3) becomes

$$
S(q)=4 \pi a_{x} a_{x} a_{z} \frac{\sin (K)-K \cos (K)}{K^{3}}
$$

where $K=\sqrt{\left(a_{x} q_{x}\right)^{2}+\left(a_{x} q_{y}\right)^{2}+\left(a_{z} q_{z}\right)^{2}}$. Substituting (20) into (8), $d$ is

$$
d\left(x, y ; q_{z}\right)=\frac{2}{q_{7}} \sin \left(q_{z} a_{z} \sqrt{1-\left(\frac{r}{a_{x}}\right)^{2}}\right) H\left(a_{x}-r\right)
$$

where $r$ is the radial length on the $x-y$ plane. Consequently, from (10a) with $N=0$, 


$$
u(x, y)=a_{z} \sqrt{1-\left(\frac{r}{a_{x}}\right)^{2}} H\left(a_{x}-r\right)
$$

which is exactly the upper surface of the spheroid and the lower surface can be obtained by using (10b).

\section{Theory for Finite Spatial-Frequency Bandwidth}

The bandlimited frequency domain (the three-dimensional shape factor q-space) is considered to be a spherical shell with $\mathrm{q}_{\mathrm{a}}$ and $\mathrm{q}_{\mathrm{b}}$ as the inside and outside radii respectively. In general, the interception between this spherical shell and the infinite hyper-plane $q_{z}=$ constant, $D(q)$, can be either an annular disk, or a circular disk depending on whether $q_{z}<q_{a}$, or $q_{a} \leq q_{z} \leq q_{b}$. In this paper, $D(q)$ is chosen to be at $q_{z}=q_{a}[7]$ where the circular disk has the largest size, as is shown in Fig. 3. With such a choice, $\mathrm{D}(\mathbf{q})$ becomes

$$
D(q)=\left\{q \mid\left[q_{z}=q_{a}\right] \cap\left[\left(q_{x}\right)^{2}+\left(q_{y}\right)^{2} \leq\left(q_{D}\right)^{2}\right]\right\}
$$

where $q_{D}=\sqrt{q_{b}^{2}-q_{a}^{2}}$ is the radius of $D(q)$. Equivalently, the window function $K(q)$ for $D(q)$ is

$$
K(q)=\delta\left(q_{z}-q_{a}\right) H\left(q_{D}-\sqrt{q_{x}^{2}+q_{y}^{2}}\right)
$$

where $\delta\left(q_{z}-q_{a}\right)$ is the Dirac delta function. The corresponding Fourier transform, $k(x)$, of $K(q)$ is

$$
\begin{aligned}
k(x) & =\frac{1}{(2 \pi)^{3}} \int_{-\infty}^{\infty} \int_{-\infty}^{\infty} \int_{-\infty}^{\infty} K(q) e^{-i q \cdot x} d q \\
& \approx \frac{1}{2 \pi} \delta(x) \delta(y) e^{-i q_{a} z}
\end{aligned}
$$

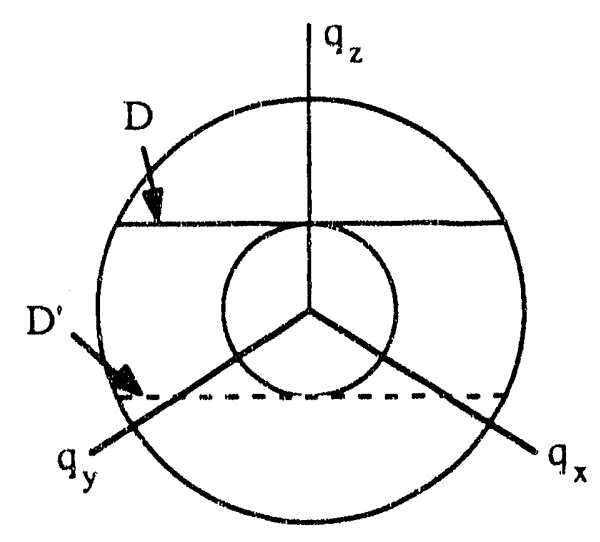

Figure 3. The data disk $D(q)$ and its image $D^{\prime}(q)$ in the spherisal shell whose inside and outside radii are $q_{a}$ and $q_{b}$ respectively. 
where $q_{b}$ is assumed to be sufficiently large [7]. Applying this pair of window functions, $k(x)$ and $K(q)$, to the Fourier transform pair, $\gamma(x)$ and $S(q)$ from Eq. (3), one has

$$
g\left(u, v ; q_{a}\right)=d_{D}\left(x, y ; q_{a}\right)
$$

where the measurable set $d_{D}\left(x, y ; q_{a}\right)$ is obtained from the disk $D(q)$

$d_{D}\left(x, y ; q_{a}\right)=\frac{1}{(2 \pi)^{2}} \iint_{D(q)} S\left(q_{x}, q_{y} ; q_{a}\right) e^{-i\left(q_{x} x+q_{y} y\right)} d q_{x} d q_{y}$.

Since Eq. (27) is similar to (8), the surfaces $u$ and $v$ can be determined by the previous analysis.

Thus,

$$
\begin{aligned}
& u=\frac{1}{\mathrm{q}_{\mathrm{a}}}\left[\tan ^{-1} \frac{\operatorname{Im}\left(\mathrm{d}_{\mathrm{D}}\right)}{\operatorname{Re}\left(\mathrm{d}_{\mathrm{D}}\right)}+\sin ^{-1} \frac{\mathrm{q}_{\mathrm{a}}\left|\mathrm{d}_{\mathrm{D}}\right|}{2}+N_{\mathrm{u}} \pi\right] \\
& \mathrm{v}=\frac{1}{\mathrm{q}_{\mathrm{a}}}\left[\tan ^{-1} \frac{\operatorname{Im}\left(\mathrm{d}_{\mathrm{D}}\right)}{\operatorname{Re}\left(\mathrm{d}_{\mathrm{D}}\right)}-\sin ^{-1} \frac{\mathrm{q}_{\mathrm{a}} / \mathrm{d}_{\mathrm{D}}}{2}+\mathrm{N}_{\mathrm{v}} \pi\right]
\end{aligned}
$$

where $N_{u}, N_{v}=0, \pm 1, \pm 2, \pm 3, \ldots$. Obviously when $u=-v$, results similar to Eq. (10) can be obtained. To determine the appropriate values of the ambiguous constants $N_{u}$ and $N_{v}$, future numerical studies are needed.

Another method of analysis for the finite bandwidth case is to replace the Heaviside function, as is shown in Eq. (24), by a Gaussian function in the window function $K(q)$. In this approach, no assumption of high frequency approximation is required. This new window function $\mathrm{K}_{\mathrm{G}}(\mathbf{q})$ is given as

$$
K_{G}(q)=\delta\left(q_{z}-q_{a}\right) e^{-\left(q_{x y} /\left(p q_{D}\right)\right)^{2}}
$$

where $q_{x y}=\sqrt{q_{x}^{2}+q_{y}^{2}}$ and $p$ is an arbitrary ncizero positive real number. Since in practice a Gaussian function must be truncated at the edge of the disk $D(\mathbf{q})$, the parameter $p$ is thus included to concrol the amplitude at the truncation. For this new window function, its Fourier transform $\mathrm{k}_{\mathrm{G}}(\mathbf{x})$ is [7]

$$
\begin{aligned}
k_{G}(x) & =\frac{1}{(2 \pi)^{3}} \int_{-\infty}^{\infty} \int_{-\infty}^{\infty} \int_{-\infty}^{\infty} K_{G}(q) e^{-i q \cdot x} d q \\
& =\frac{1}{2 \pi} e^{-i q_{a} z} \frac{1}{4 \pi}\left(p q_{D}\right)^{2} e^{-(1 / 4)\left(r_{x y} p q_{D}\right)^{2}}
\end{aligned}
$$


where $r_{x y}=\sqrt{x^{2}+y^{2}}$. Applying this pair of window functions, $k_{G}(x)$ and $K_{G}(q)$, to the Fourier transform pair, $\gamma(\mathbf{x})$ and $S(q)$ from Eq. (3), one has

$$
\mathrm{g}\left(\mathrm{u}, \mathrm{v} ; \mathrm{q}_{\mathrm{a}}\right)=\mathrm{d}_{\mathrm{D}}^{\mathrm{W}}\left(\mathrm{x}, \mathrm{y} ; \mathrm{q}_{\mathrm{a}}\right)
$$

where the measurable function $\mathrm{d}_{\mathrm{D}}^{\mathrm{W}}\left(\mathrm{x}, \mathrm{y} ; \mathrm{q}_{\mathrm{a}}\right)$ is the two-dimensional inverse Weierstrass transformation [10] of the data set $d_{D}^{G}\left(x, y ; q_{a}\right)[7]$ and

$$
\begin{aligned}
& d_{D}^{W}\left(x, y ; q_{a}\right)=\frac{-1}{4 \pi} \int_{\sigma_{y^{\prime \prime}-i \infty}}^{\sigma_{y^{\prime}+i \infty}} \int_{\sigma_{x^{\prime}-i \infty}}^{\sigma_{x^{\prime}+i \infty}} d_{D}^{G}\left(x^{\prime}, y^{\prime} ; q_{a}\right) e^{\left[\left(x^{\prime}-x\right)^{2}+\left(y^{\prime}-y\right)^{2}\right] / 4} d x^{\prime} d y^{\prime} \\
& d_{D}^{G}\left(x, y ; q_{a}\right)=\frac{1}{(2 \pi)^{2}} \int_{-\infty}^{\infty} \int_{-\infty}^{\infty} S\left(q_{x}, q_{y} ; q_{a}\right) e^{-\left(q_{x y} /\left(p q_{D}\right)\right)^{2}} e^{-i\left(q_{x} x+q_{y} y\right)} d q_{x} d q_{y}
\end{aligned}
$$

with $\sigma_{x^{\prime}}$ and $\sigma_{y^{\prime}}$ being real variables [10]. Since Eq. (32) is similar to (8), the surfaces $u$ and $v$ can be determined by the previous analysis. Thus,

$$
\begin{aligned}
& u=\frac{1}{q_{a}}\left[\tan ^{-1} \frac{\operatorname{Im}\left(d_{D}^{W}\right)}{\operatorname{Re}\left(d_{D}^{W}\right)}+\sin ^{-1} \frac{q_{a}\left|d_{D}^{W}\right|}{2}+N_{u} \pi\right] \\
& v=\frac{1}{q_{a}}\left[\tan ^{-1} \frac{\operatorname{Im}\left(d_{D}^{W}\right)}{\operatorname{Re}\left(d_{D}^{W}\right)}-\sin ^{-1} \frac{q_{a}\left|d_{D}^{W}\right|}{2}+N_{v} \pi\right]
\end{aligned}
$$

where $N_{u}, N_{v}=0, \pm 1, \pm 2, \pm 3, \ldots$ Again when $u=-v$, results similar to Eq. (10) can be obtained. To determine the appropriate values of the ambiguous constants $N_{u}$ and $N_{v}$, future numerical studies are needed.

\section{Discussion and Conclusion}

This paper has proposed a surface reconstruction technique that requires only two-dimensional hyper-plane data in the spatial-frequency domain (q) and thus significintly improves on the traditional three-dimensional approach [3]. For the above studies, whether with infinite or finite frequency spectra, all the algorithms share a similar expression as can be seen in Eqs. $(y),(28)$ and (34). The differences are found in the selection of the hyper-plane $q_{z}=$ constant and in the data processing of the shape factor set $S(q)$ to acquire the appropriate "data function" $d\left(x, y ; q_{z}\right)$, $d_{D}\left(x, y ; q_{a}\right)$, or $d_{D}^{W}\left(x, y ; q_{a}\right)$. In general, this technique is ill-posed, as is shown in Eqs. (9), (28) and (34), which is inherent with most inverse problems. The numerical instability in this algorithm, however, is identified with the nonsymmetry of the surface of a general target. Hence, whenever a scatterer has a two-fold symmetric surface with respect to the assigned plane, $z=$ constant, the algorithms become stable. The validity of this method has been demonstrated by three analytical examples. 


\section{Acknowledgment}

The author is thankful to Dr. K. L. Telschow, Mr. B. A. Barna, Dr. V. G. Kogan and Prof. L. W. Schmerr for their encouragement and discussions of the present work. This work was sponsored by the U.S. Department of Energy, Office of Energy Research, Office of Basic Energy Sciences. The initial work was performed at the Ames Laboratory under DOE Contract No. W7405-ENG-82; later work was performed at the Idaho National Engineering Laboratory under DOE Idaho Field Office Contract No. DE-AC07-761D01570.

\section{References}

1. Gubernatis, J.E.; Domany, E.; Krumhansl, J.A.; Hubermann, M.: The Born approximation in the theory of the scattering of elastic waves by flaws, J. of Appl. Phys., 48 (1977) 2812-2819.

2. Hudson, J.A.; Heritage, J.R.: The use of the Born approximation in seismic scattering problems, Geophys. J. Royal Astron. Soc., 66 (1981) 221-240.

3. Koo, L.S.; Wormley, S.J.; Hsu, D.K.; Thompson, D.O.: Fourier method approaches to three-dimensional ultrasonic flaw reconstruction, in Review of Progress in QNDE, edited by D.O. Thompson and D.E. Chimenti (Plenum Press, New York), 10A (1991) 67-74.

4. Lewis, R.M.: Physical optics inverse diffraction, IEEE Trans. on Ant. and Prop., 17 (1969) 308-314.

5. Rose, J.H.: Elastic wave inverse scattering in nondestructive evaluation, Pure and Appl. Geophys., 131 (1989) 715-739.

6. Chen, J.S.: Elastodynamic ray theory and asymptotic methods for direct and inverse scattering problems, Ph.D. dissertation, Iowa State Univ., 1987.

7. Koo, L.S.: Theoretical surface reconstruction of a three-dimensional ultrasonic flaw based on the Born approximation, to appear.

8. Kogan, V.G.; Lopes, E.F.: On the Born approximation for weak uniform scatterers, Inverse Prob., 1 (1985) 331-338.

9. Vogel, C.R.: Numerical solution of a nonlinear ill-posed problem arising in inverse scattering, Inverse Prob., 1 (1985) 393-403.

10. Zemanian, A.H., Generalized Integral Transformations. New York: Dover Publications 1987.

\section{DISCLAIMER}

\footnotetext{
This report was prepared as an account of work sponsored by an agency of the United States Government. Neither the United States Government nor any agency thereof, nor any of their employees, makes any warranty, express or implied, or assumes any legal liability or responsibility for the accuracy, completeness, or usefulness of any information, apparatus, product, or process disclosed, or represents that its use would not infringe pri ately owned rights. Reference herein to any specific commercial product, process, or service by trade name, trademark, manufacturer, or otherwise does not necessarily constitute or imply its endorsement, recommendation, or favoring by the United States Government or any agency thereof. The views and opinions of authors expressed herein do not necessarily state or reflect those of the United States Government or any agency thereof.
} 

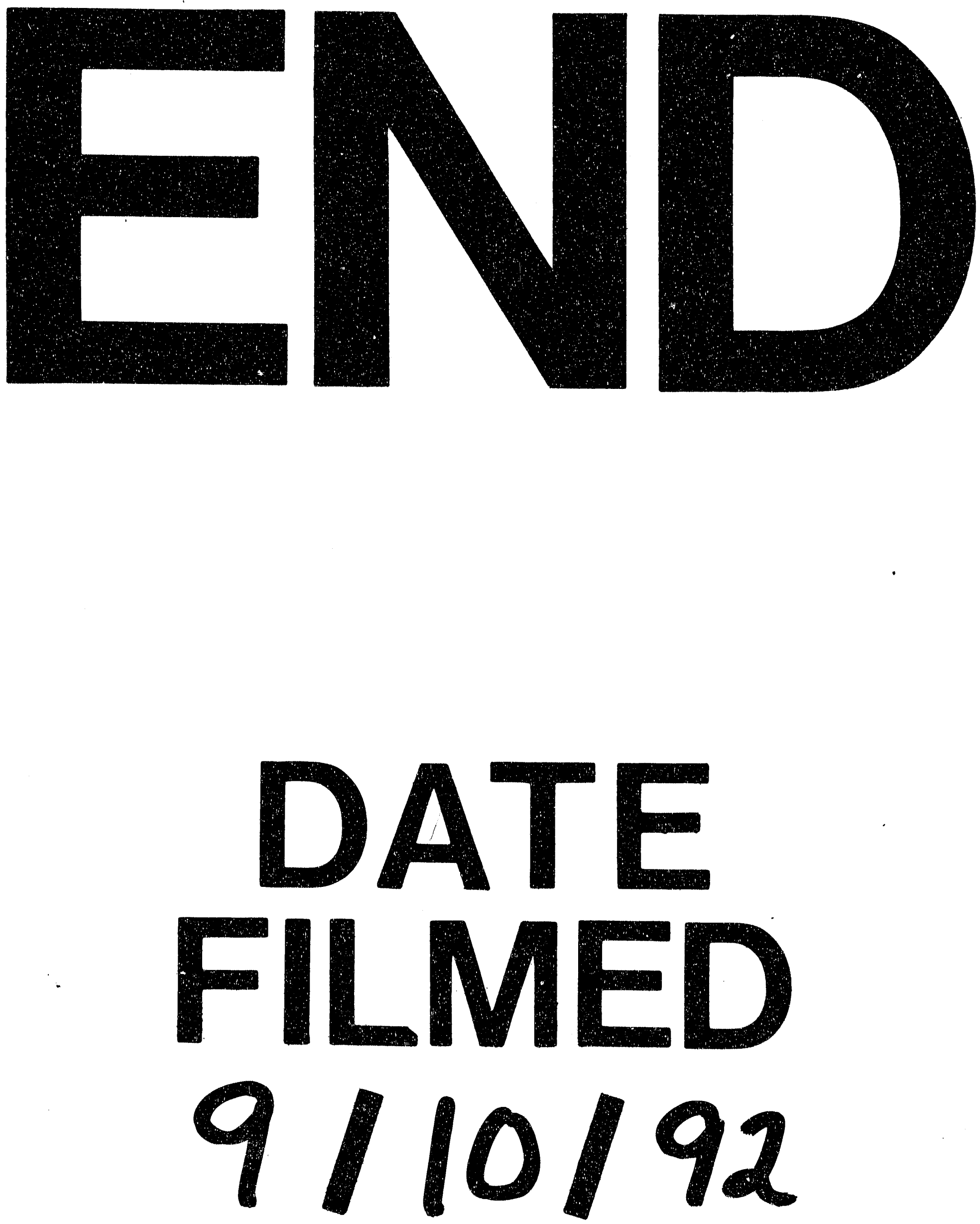
. 